\title{
LES COMMENTAIRES JOURNALISTIQUES SUR LE SPORT ONT-ILS UN SENS ?
}

\author{
Fabien Ohl ${ }^{1}$
}

Les sports médiatisés présentent deux caractéristiques singulières qui constituent des avantages majeurs sur les marchés des médias. D'une part, ils facilitent la célébration des individus et des identités plus collectives (nationales, locales ou sexuelles) et, d'autre part, ils ont l'avantage d'être assez "technicisés" pour ressembler à un spectacle universel. Le geste sportif, rationalisé, répété, contrôlé et préparé, s'est fortement éloigné de l'emprise des cultures locales ou nationales. Les sports s'accommodent assez facilement des contraintes médiatiques et se sont transformés eu égard aux contraintes télévisuelles ${ }^{2}$. Techniques, règlements, tenues, tactique, horaires ou préparation physique s'adaptent aux impératifs des marchés. Paradoxalement, les sports les plus spectacularisés sont entrés dans les logiques de consommation de produits standardisés ${ }^{3}$

1 Professeur à l'Université Marc Bloch - Strasbourg, Directeur du Laboratoire de recherche "Activités physiques et sportives et sciences sociales".

2 Les transformations des règles, des tenues, des épreuves sportives répondent à la demande des télévisions. Voir G. VIGARELlo, Techniques d'hier et d'aujourd'hui, Éd. EPS, 1988.

3 S'inscrivant ainsi dans une sorte de McDonaldization de la société ; voir G. RITZER, The McDonaldization Thesis, London, Sage, 1998.

Recherches en communication, $\mathrm{n}^{\circ} 15,(2001)$. 
tout en conservant la capacité à rappeler et à célébrer des identités spécifiques. Le plus médiatisé des sports, le football, peut être un élément essentiel, avec le tango, de la construction de "l'image du national" en Argentine, entrer dans les fêtes traditionnelles comme les navétanes au Sénégal ou encore être un moyen d'affirmer une identité communautaire dans un pays meurtri comme le Liban'.

Le succès du sport doit beaucoup à son adaptation aux modifications structurelles de l'offre médiatique ${ }^{2}$. La diffusion de la télévision, la concurrence entre les chaines de télévision et, en conséquence, la recherche de taux d'audience élevés, ont favorisé la diffusion de spectacles sportifs. Les coûts de production des spectacles sportifs et la facilité à les programmer expliquent partiellement leur usage médiatique. Les facteurs socio-économiques qui structurent l'offre ne peuvent pourtant pas expliquer à eux seuls les raisons de l'importante médiatisation des spectacles sportifs. Si les récits sportifs connaissent un engouement important, c'est à la fois parce qu'ils constituent une consommation de masse, le sport en tant que fait omnibus ${ }^{3}$ étant un bon produit marchand, et qu'ils permettent de donner du sens à un spectre assez large de conduites humaines. La forte diffusion et parfois même la fascination pour la narration sur le sport restent à comprendre. Que les enjeux objectifs des choix sociaux, politiques ou économiques soient l'occasion de nombreux commentaires pour en décoder le sens ne surprend pas. Les enjeux du sport semblent a priori plus négligeables, pourtant, s'ils ont une si grande importance c'est que sous l'apparence trompeuse de bavardages insignifiants, ils constituent aussi un gisement de significations.

Nous proposons donc de voir que le commentaire sportif n'est pas un simple bavardage, qu'il constitue un récit d'autant plus attractif qu'il comporte une dimension "fatale". Nous voudrions montrer que la lisibilité et la simplicité des interactions sportives permettent à la fois aux narrations sportives de s'adapter aux logiques de l'offre marchande et de donner du sens à des actions situées dans d'autres cadres sociaux. Le sport constitue ainsi une façon de mettre en scène et de débattre des façons légitimes d'agir. Notre travail se base essentiellement sur l'analyse de contenu des articles du journal Le Monde ;

\footnotetext{
1 Voir par exemple les intéressantes contributions au numéro 7 de la revue Sociétés et représentations (décembre 1998) sur le thème "football et sociétés".

2 Voir D. MARCHETTI, "Le football saisi par les médias", Sociétés et représentations, $\mathrm{n}^{\circ}$ 7, déc. 1998, pp. 309-331.

3 P. Bourdieu, Sur la télévision, Paris, Liber, 1996, p. 16.
} 
une étude complémentaire d'articles pris dans d'autres journaux permet à la fois d'observer des éléments relativement stables et une diversité de commentaires selon les sports et les journaux ${ }^{1}$.

\section{Le commentaire sportif : un bavardage sur des faits omnibus ?}

Les modifications structurelles de l'offre médiatique ne suffisent pas à comprendre pourquoi le sport connaît un tel engouement ${ }^{2}$. Le sport, qui se targue de sa gratuité et d'une éthique du désintéressement, peut, à certains égards, s'apparenter à un gaspillage, une activité inutile, bref, à une dissipation d'énergie en pure perte. Dans le sens commun et dans certaines analyses, parler des faits divers et du sport semble correspondre à un bavardage dénué de signification ${ }^{3}$. Le discours sur le sport est donc souvent l'occasion d'évoquer de nombreux lieux communs qui ont d'abord une fonction phatique. Le bavardage est assez neutre ; très souvent, en effet, commenter un spectacle sportif est une façon de réaffirmer des liens sociaux ${ }^{4}$ ou de

1 L'échantillon de textes sur lequel portent nos analyses est constitué de 890 articles publiés en 1996 dans le journal Le Monde (football $\mathrm{n}=368$, cyclisme $\mathrm{n}=186$, voile $\mathrm{n}=160$, tennis $\mathrm{n}=176$ ) et de 50 articles d'autres journaux. Bien que l'on observe une certaine homogénéisation de la presse sportive (D. MARCHETTI, op. cit.), ce choix ne prétend pas être représentatif du journalisme sportif. Il est évident que les articles du Monde sont plus narratifs et moins emphatiques que ceux de $L^{\prime} E$ quipe et moins descriptifs que les commentaires des quotidiens régionaux. Une méthode d'analyse de données textuelles (occurrence de mots et de phrases) nous a permis des comparaisons quantitatives. Selon la mệme méthode, les commentaires sportifs ont été comparés avec des articles portant sur une autre activité culturelle (musique rock, $n=182$ ). Même si les analyses quantitatives du langage sont assez pauvres par rapport au sens des textes, c'est un travail qui a facilité les analyses qualitatives du contenu des articles.

2 Selon Médiamétrie et Eurodata T.V. (Une année de télévision dans le monde, étude réalisée en 1996 à partir d'un échantillon de quarante pays), le sport figure au palmarès des 10 meilleures audiences annuelles dans quatre pays sur cinq et à la première place dans 13 pays. Les meilleurs taux d'audience sont liés à l'implication d'une équipe nationale. Source : La Lettre de l'économie du sport, $n^{\circ} 392$, mai 1997, p. 4. Pendant l'année 1996, les sports sont arrivés en tête de l'audimat au Danemark avec le handball, en Finlande avec le hockey sur glace et en Allemagne, Grèce, Pays-Bas, Italie et Turquie avec le football.

3 Umberto Eco parle d'un bavardage sportif qui a pour seule fonction de permettre la communication. Voir U. ECO, La guerre du faux, Paris, Grasset, 1985.

4 L'analyse, par J.-M. Faure, d'une photo prise lors d'un match de football rappelle l'importance du commentaire : "L'instant délicat du pénalty. Tous se rassemblent et entourent le joueur pour "commenter» la force du coup de pied" (J.-M. FAURE, 
faire étalage de ses compétences dans la sémantique sportive. Le temps qu'il fait, la mort de la Princesse Diana, l'insécurité, les résultats d'une rencontre, l'oreille arrachée du boxeur E. Holyfield, etc. sont des ressources sûres des interactions sociales et permettent une communication peu risquée (le consensus ne met pas en jeu les faces des interactants).

Dans cette perspective, les classements, la désignation de la meilleure équipe comme la sélection des athlètes méritant de figurer au Panthéon du sport, ressemblent à des palabres aussi interminables que vides de sens. Le sport ne choque pas et fait souvent le consensus. Il rassemble plus qu'il ne divise, paraît ne toucher à rien d'essentiel et évite de poser des questions pertinentes sur des sujets plus importants. Les narrations des événements sportifs sont à rapprocher des faits divers ; d'ailleurs la place laissée aux faits divers et au sport dans les colonnes des quotidiens évolue dans le même sens ${ }^{1}$. Comme le suggère Pierre Bourdieu, le sport est un fait omnibus. Loisir approprié à tous les âges, pratique éducative, activité favorable à l'hygiène, les qualificatifs dominants associés aux pratiques sportives laissent peu de place à de rares disqualifications qui portent essentiellement sur les excès de la pratique de compétition (le dopage par exemple) et rarement sur la pratique elle-même. On comprend d'ailleurs que l'association des sports et des faits divers corresponde à la situation idéale d'un point de vue de la construction de l'événement journalistique. Les affaires O. J. Simpson, Mike Tyson², Éric Cantona, Bernard Tapie, les "rescapés" de La Vanoise ou les problèmes de hooliganisme garantissent une bonne audience télévisuelle et un bon tirage des journaux ${ }^{3}$.

"Les fouteux de Voutré", Actes de la recherche en sciences sociales, Paris, $\mathrm{n}^{\circ} 80$, nov. 1989, pp. 68-73.

1 Par exemple, le nombre d'articles publiés par le journal Le Monde dans les rubriques sport et faits divers évolue dans le même sens. Voir F. OHL, "The Sport in Newspapers as Comments on Everyday Life", in XIVe Congrès mondial de sociologie, Montréal, 1998.

2 Les écarts de conduite du boxeur Mike Tyson font la "une" des journaux. Le fait divers le plus récent a eu lieu le samedi 28 juin 1997. Durant le championnat du monde de boxe, il arrache de ses dents l'oreille de son rival Evander Holyfield. C'est d'ailleurs l'occasion de rappeler qu'on ne se défait pas de sa vraie nature (entre autres titres: "«Iron» Mike retrouve sa vraie nature", A. MERCIER, Le Monde, 1er juill. 1997, p. 22 et M. CHEMIN, "Tyson met la boxe Chaos", Libération, ler juill. 1997).

3 Par exemple, l'association sport et fait divers (Lundi 2 mai 1994, "La mort au tournant" [à la "une"], Formule 1 : Senna et Ratzenberger se tuent au GP de Saint- 


\section{Le commentaire sportif présente les caractéristiques d'un récit}

Les sports ont surtout une place importante dans la vie des populations masculines et des moins de $20 \mathrm{ans}^{1}$. L'engouement pour le récit sportif n'est pas nouveau ; il résiste aux transformations des techniques de diffusion et profite même de la montée en puissance de l'image ${ }^{2}$.

Bien qu'il ne s'agisse pas d'éléments spécifiques, les spectacles sportifs cumulent de nombreuses caractéristiques qui en font des événements capables de générer facilement des récits. En effet, il y a dans la plupart des cas une unité de temps (durée d'une rencontre) et de lieu (stade, mer, rivière, rocher...), et les acteurs sont souvent perçus comme étant héroïques. La simplicité des schémas proposés par les spectacles sportifs et l'incertitude des résultats des événements favorisent l'émergence d'intrigues aisément perceptibles. L'intérêt pour le lecteur est d'autant plus vif que le journaliste est un intermédiaire culturel qui donne la parole, en la transformant parfois, à des personnes qui en sont souvent dépossédées parce qu'exclues du champ médiatique et de la culture ${ }^{3}$.

Marin) a assuré la deuxième meilleure vente de L'Équipe (723 912 exemplaires) et le pourcentage le plus faible d'invendus $(8,2 \%)$ entre 1988 et 1994 (source : J.F. BourG, J.-J.GougueT, Analyse économique du sport, Paris, PUF, 1998). On peut noter aussi que les avocats de l'affaire O. J. Simpson animent des émissions de télévision aux États-Unis.

1 Les enquêtes de l'INSEE $(1984,1988)$, du ministère de la Culture $(1981,1997)$, de l'INSEP (1985) ou l'étude du lectorat des journaux et rubriques sportives en atteste. Par exemple, le lectorat de L'Équipe est composé de $86 \%$ d'hommes et ne se féminise pas malgré les tentatives (source : La Lettre de l'économie du sport, $n^{\circ} 340,20$ mars 1996).

${ }^{2}$ En l'absence de diffusion télévisuelle, les articles fabriquaient du récit (voir G. VIGARELLO, "Un show quasi universel, les métamorphoses du spectacle sportif", Esprit, n $^{\circ} 4$, avril 1987, pp. 159-167). Aujourd'hui, les commentaires de la presse continuent à fabriquer du récit en préparant la perception de l'image, en décodant les logiques de l'action et en prolongeant le commentaire emphatique du direct.

3 C'est un des éléments de l'argumentation de J. S. LeIre LOPES et S. MARESCA, "La disparition de la joie du peuple. Notes sur la mort d'un joueur de football", Actes de la recherche en sciences sociales, $\mathrm{n}^{\circ} 79$, sept. 1989, pp. 21-36, pour expliquer la passion de nombreux Brésiliens pour Garrincha, footballeur vedette des années 50. Des arguments identiques peuvent aussi expliquer d'autres succès télévisuels ; c'est par exemple le cas de L'inspecteur Derrick (la série télévisée non américaine qui se vend le mieux dans le monde). Herbert Reinecker, auteur du scénario déclare (cité 
Si le bavardage sportif est bien un fait omnibus qui semble tout à fait accessoire eu égard à un journalisme qui se voudrait plus critique, le langage du journaliste prend néanmoins du sens parce qu'il contribue à observer des faits de société semblant insignifiants. Le "vide" apparent des commentaires est obéré de sens. En effet, les reportages télévisés ou les articles de presse proposent des récits qui mettent en scène une diversité de conduites et de codes. Les thèmes dominants ont été analysés et dénoncés ${ }^{1}$, mais il nous semble que l'abondance des pratiques sportives et la diversité des analyses s'inscrivent sur des registres plus larges de l'action. C'est aussi dans le traitement quotidien d'événements ordinaires que se façonne le sens. En rappelant les règles auxquelles sont soumises les conduites, les paroles les plus insignifiantes participent à une mise en sens qui est également une mise en ordre ${ }^{2}$. Les enjeux sportifs sont certainement secondaires par rapport à d'autres domaines traités en priorité par la presse (politique, finance, économie, éducation...) mais le sport et ses mises en scène contribuent aussi à l'organisation symbolique du social ${ }^{3}$.

dans Le Monde par L. Delattre, 23-24 févr. 1997, p. 2) que "c'est le triomphe de l'ordre humain sur le crime incurable". Ce qui semble plaire à une partie des auditeurs, c'est à la fois le caractère très ordinaire et les dimensions morales du personnage (pas de sexe, d'alcool ni de politique).

1 Le thème dominant est évidemment celui de la guerre, les affrontements sportifs étant des simulacres de batailles et le sport et la guerre assurant une promotion de l'hégémonie masculine; voir notamment J.-M. BRöHM, Les meutes sportives. Critiques de la domination, Paris, L'Harmattan, 1993. La métaphore guerrière se prête particulièrement bien à une mise en récit ; la communication sur le sport n'en a pas l'exclusivité. Elle constitue aussi l'un des principaux réseaux métaphoriques de la communication politique (voir G. GAUTHIER, "La métaphore guerrière dans la communication politique", Recherches en communication, $\mathrm{n}^{\circ} 1,1993, \mathrm{pp} .131-146$. Par ailleurs, c'est plus généralement le système métaphorique «la discussion = la guerre», qui semble fortement ancré dans notre culture; voir G. LAKOFF, M. JOHNSON, Les métaphores dans la vie quotidienne, Paris, Éd. de Minuit, 1983.

2 Au sens de E. GoFmMAN, Les rites d'interaction, Paris, Éd. de Minuit, 1974, qui indique que "l'ordre social tire sa substance d'un ensemble de petits comportements disciplinés" et pour qui les paroles "vides" sont souvent chargées de significations.

3 Le sport apparaît comme un symptôme ou un palliatif d'une crise du symbolique, de la représentation et du social ; voir M. AUGÉ (entretien avec G. DERÈzE), "Le sport comme phénomène surmoderne", Recherches en communication, $n^{\circ} 5,1996$, p. 171. Même le domaine de l'économie du sport n'échappe pas aux logiques symboliques. Ainsi, l'efficacité de certaines entreprises sportives de production de biens ou de spectacles sportifs est à la fois symbolique et économique. Bien que les enjeux économiques du sport ne soient pas négligeables, ils sont plus limités que l'on ne pourrait le croire. Par exemple, les marques Nike et Adidas ont une très forte notoriété compte tenu de leurs chiffres d'affaires. Selon l'enquête Teen 
L'insignifiance apparente des commentaires sportifs ne doit pas dissimuler le fait que les rhétoriques sont chargées de sens ${ }^{1}$. C'est le cas de la centaine de radios américaines consacrées au bavardage sur le sport (Talk Radios) qui constituent, pour ceux qui se pensent apolitiques ou qui ne se sentent pas libres d'exprimer leurs opinions politiques, un espace d'expression très marqué politiquement (hommes, conservateurs républicains, catégories moyennes et basses...) $)^{2}$. Les récits sportifs offrent la possibilité de donner du sens aussi bien à des conduites individuelles qu'à des attentes collectives. Si de nombreux travaux sociologiques ou historiques se sont intéressé aux liens entre le spectacle sportif et la célébration d'une collectivité à travers un succès ou une place d'honneur (la victoire des Bleus en Coupe du Monde en étant un exemple frappant), le flot de commentaires apparemment insignifiants, notamment les menus propos sur le quotidien de la vie sportive (les amitiés, les souffrances, l'honneur, la vie de famille, les sentiments...) expriment également des conceptions de la vie en société. En tentant de décrire, d'analyser et d'interpréter un certain nombre d'actions, ils débattent des manières d'agir et d'interagir dans le sport et dans la société. Les commentaires des faits et gestes des acteurs du sport constituent donc d'intéressants matériaux d'analyse.

Generation 1997 (citée par Sport Première, n 174, mars-avril 1998, p. 113), Nike et Adidas sont en tête des marques préférées des adolescents. Leurs chiffres d'affaires 1997 (respectivement 55 et 22 milliard de francs) sont relativement faibles eu égard à d'autres entreprises : la notoriété internationale du groupe Adidas-Salomon est bien supérieure à celle de Renault qui pourtant pèse dix fois plus (en termes de CA avant l'acquisition de Nissan). L'économie du sport semble même servir de modèle aux autres secteurs ; $\mathrm{H}$. Juvin ("La loi des champions", Le Monde, 13 avril 1999, p. IV) affirme que: "dans l'industrie comme dans les services, de nombreux secteurs sont entrés dans une logique de compétition et de vedettariat qui tire ses lois du spectacle et du sport".

1 Sans intention avérée, les commentaires sportifs véhiculent des stéréotypes raciaux en rappelant par exemple, à travers des propos insignifiants, les clichés sur les qualités physiques des noirs et psychologiques des blancs. Voir D. MCCARTHY, R. L. JONES, "Speed, Agression, Strengh and Tactical Naïveté. The Portrayal of the Black Soccer Player at Television", Journal of Sport \& Social Issues, vol. 21, $\mathrm{n}^{\circ} 4$, Nov. 1997, pp. 348-362.

2 D. T. GoldBerG, "Sports, Talk Radio and the Death of Democracy", Journal of Sport \& Social Issues, vol. 22, n 2, May 1998, pp. 212-223. 


\section{Le sport en tant qu'activité "fatale"}

Les caractéristiques des sports facilitent leur mise en scène. Les activités sportives restent un moment privilégié de libération et d'expression publique des émotions "d'excitation" (exitement) ${ }^{1}$. Le spectacle sportif est constitué de situations fatales qui permettent des évaluations morales et sociales ${ }^{2}$. Les affrontements non simulés des spectacles sportifs renvoient aussi à d'autres situations par une sorte de "mimèsis" ; pour N. Elias et E. Dunning, le sport "imite de manière sélective un affrontement de la vie réelle"3. Les spectacles sportifs et leurs rituels ${ }^{4}$, en mettant en scène des circonstances fatales, favorisent la lisibilité des valeurs de l'action d'un individu ou d'un groupe $^{5}$. Les jeux et les sports impliquent des engagements qui nécessitent souvent de porter les efforts à la limite pour l'emporter. Même si, à l'évidence, les conditions de la vie ordinaire ne sont pas aussi propices à une compétition aussi clairement définie ${ }^{6}$, l'énoncia- $^{2}$ tion sportive donne du sens à la vie quotidienne; les exploits, les performances hors normes comme les ratages des sportifs (les contre-

1 Il s'agit d'un processus de "controlled decontrolling of emotions" ; voir N. ELIAS, E. DUNNING, Sport et violence, Paris, Fayard, 1994.

2 Goffman note que "Si l'on prend pour base la notion de devoir fatal, on peut considérer l'action comme une sorte de mise en scène personnelle et ritualisée de l'aspect moral de l'accomplissement de ce devoir" (E. GoFFMAN, op. cit., p. 198). Même s'ils contiennent déjà une interprétation des faits, les articles de presse ont souvent été utilisés, notamment par E. Goffman, parce qu'ils relatent des interactions de la vie quotidienne.

3 N. Elias, E. DUNNING, op. cit., p. 64.

4 La ritualisation du sport semble devoir contribuer à faire des sportifs les vecteurs de l'affirmation d'importantes valeurs sociales. Voir S. BIRREL, "Sport as Ritual : Interpretations from Durkheim to Goffman, Social Forces, vol. 60, n 2, Dec. 1981, pp. 354-376.

5 Nous reprenons l'analyse de Goffman (op. cit., p. 177) : "C'est lorsque les circonstances sont visiblement fatales -lorsqu'elles tirent à conséquence et sont problématiques- et seulement en liaison étroite avec de telles circonstances, qu'un nouvel ensemble d'aptitudes apparaît" et (ibid., p. 178): "Les qualités primordiales peuvent s'exprimer dans une situation dépourvue de fatalité ; les qualités du caractère ne se révèlent que lors d'événements fatals... on peut approuver, désapprouver, ou s'abstenir de juger les qualités primordiales. Le caractère, par contre, est toujours pesé d'un point de vue moral, pour la simple raison que l'aptitude à mobiliser ses facultés dans l'instant est toujours sujette à évaluation sociale".

6 E. Goffman remarque néanmoins que "chaque jour, de bien des façons, on peut essayer de marquer des points, et chaque jour, de bien des façons, on peut dégringoler" (ibid., p. 198). 
performances) tracent les limites des cadres ordinaires de l'activité humaine. Les "skieurs de l'impossible ", "Magic Johnson ", le "Roi Pelé " ou la "Dream Team ", les pratiques extrêmes ou les grands athlètes qualifiés de "monstres" du sport rappellent les configurations et les limites des cadres primaires. A l'image des masques, des figures du grotesque et de la monstruosité abondamment observés par les anthropologues, ils fixent ou amènent à réfléchir sur les "dimensions" de la culture ${ }^{1}$. L'intérêt du public est d'autant plus vif que, contrairement à ce qui se passe en général dans la littérature, le théâtre ou le cinéma, le récit sportif se base sur des situations réelles ${ }^{2}$.

Les sports n'ont certes pas l'exclusivité de ces caractéristiques. Les faits divers, les accidents de la route, les conflits présentent des propriétés analogues. Cependant, le sport se situe essentiellement sur le registre symbolique même si dans les sports de montagne, la voile ou les courses automobiles, le jeu avec une fatalité réelle fascine les lecteurs. Les situations fatales sont nombreuses mais dépendent le plus souvent de l'actualité (guerres, sauvetages, accidents, etc.). D'autres activités sociales sont utilisées : une prise d'otage ou une guerre sont aussi captivantes à condition que leur durée soit limitée ; si les morts réelles et symboliques ne peuvent se confondre, la tension est proche. La particularité des épreuves sportives, par rapport à d'autres événements, c'est qu'elles provoquent en principe une tension et une excitation agréables qui les différencient des drames réels tels que les guerres; les récits se focalisent d'abord sur les "gagnants" alors que le malheur des "vaincus" ou les souffrances des victimes sont au premier plan du journalisme qui porte sur les faits divers ou les conflits armés.

Le spectacle sportif présente aussi l'avantage de proposer un spectre assez large de situations (individuelles, collectives, affrontements, nature...) qui peuvent renvoyer à de multiples circonstances de la vie quotidienne. Il présente cependant une certaine ambiguitté. C'est à la fois une activité mimétique que l'on peut rapprocher d'une tragédie théâtrale ou d'une fiction, puisqu'il s'agit bien d'un jeu, et une

1 E. Goffman, Les cadres de l'expérience, Paris, Éd. de Minuit, 1991 (1974), pp. 39 40.

2 Cette interprétation est confirmée par l'analyse des audiences télévisuelles. Les activités à risque, l'accident, le danger sportif, le drame ou encore l'association entre fait divers et sport attirent beaucoup le public. Voir Laurence CHAUSSON (Responsable Marketing Programmes, Eurosport), Représentation et valorisation des sports à la télévision selon les pays, appréciation selon les populations, communication orale au colloque "Montrer le sport", Paris, INSEP, mars 1999. 
activité que 1'on peut assimiler à une tragédie réelle, la victoire ou la défaite étant imprévisible et sans appel. La ligne de démarcation entre le jeu et la réalité d'une activité sociale aux enjeux économiques et symboliques réels est souvent incertaine.

Ce sont aussi les caractéristiques propres du sport qui facilitent son utilisation. Les dimensions importantes du caractère (intégrité, courage, sang-froid, mérite, cran...) analysées par E. Goffman sont abondamment mises en scène dans les sports. L'intégrité (définie comme propension à résister aux tentations) est très souvent théâtralisée parce qu'elle participe au maintien de l'ordre cérémoniel. La référence rituelle au fair-play correspond au rappel et à la mise en scène des règles de déférence. Elle assure un respect des faces et une sacralisation de la personne ou des institutions. Par exemple, le sang-froid ${ }^{1}$ est fréquemment valorisé dans le sport pour deux raisons principales. D'une part, se comporter en tant qu'interactant compétent est important pour tous ; l'agitation disqualifie l'interactant et est une menace pour l'ordre de l'interaction ${ }^{2}$. D'autre part, l'intelligence et l'habileté physique sont des qualités nécessaires à l'accomplissement de la plupart de nos tâches quotidiennes, qu'elles impliquent ou non une co-présence. Il est donc normal que "l'aptitude à entretenir celles-ci au milieu des difficultés soit universellement encouragée "3. Les épreuves sportives mettent en scène des capacités de contrôle de soi et rappellent que les règles sociales doivent être respectées. Les liens "normaux" entre les conduites et les codes sont au cœur des commentaires sportifs.

1 "Le sang-froid apparaît comme une préoccupation et une valeur commune à de nombreuses cultures et couches sociales différentes" (E. GoFFMAN, Les rites..., op. cit., p. 189). La question du sang-froid rappelle que l'affaiblissement des rites sociaux traditionnels (mariages, enterrements, carnavals...) a favorisé l'extériorisation des émotions par des rituels sportifs (N. ELIAS, E. DuNNING, op. cit., p. 85), le sport présentant l'avantage de pouvoir mettre en scène un contrôle de soi tout en tolérant des écarts liés aux états émotionnels.

2 L'ordre social maintenu au sein de la réunion tire ses matériaux, sa substance, d'une somme de comportements disciplinés" (E. GoFFMAN, Les rites..., op. cit., p. 187).

3 Ibid., p. 188.

4 Par exemple : "L'ITF confirme la victoire du Brésil en Coupe Davis, il faut savoir garder son sang-froid un peu plus que d'habitude. Nous espérons que ces sanctions financières serviront d'exemple aux cent vingt-sept nations qui doivent participer à la Coupe Davis en 1997. Les règles et les traditions de l'épreuve doivent être respectées" (B. MATHIEU, Le Monde, 9 oct. 1996, p. 21). 
La valorisation du sang-froid, de l'intelligence et des habiletés physiques dans les événements sportifs sert de modèle aux conduites en société. La fréquence des bilans réalisés après les grands événements traduit le souci de tirer une morale des engagements des acteurs ${ }^{1}$. Ce ne sont pas les seules qualités retenues par Goffman ${ }^{2}$ que le sport permet de mettre en scène. Les traits de caractère, les problèmes économiques et sociaux, les questions éthiques, etc. sont abordés dans les récits sportifs. Malgré des caractéristiques dominantes, la diversité des pratiques, des spectacles, des mises en scène et les interprétations qu'en font les lecteurs ou les auditeurs constituent une offre narrative assez étendue pour pouvoir suggérer des morales de l'action extensibles à différentes situations de la vie quotidienne. Il s'agit d'une résonance émotionnelle; même si la vie quotidienne est généralement plus terne : la plupart des événements de loisir éveillent des émotions liées à celles que les individus expérimentent dans d'autres sphères "3. Les relations entre les mises en scène sportives et les situations de la vie quotidienne ne sont pas simples, la tonalité, l'intensité émotionnelle ou les niveaux de complexité ne sont pas systématiquement homologues : il s'agirait plutôt d'un " couplage flou "4. Les liens entre les spectacles sportifs et la vie quotidienne sont ténus ; la diversité des spectateurs des grands événements en est la preuve. Les milliards de spectateurs de sexe ou d'âge différents présentent une grande diversité culturelle. Le lien se joue davantage au niveau "des sentiments suscités par des événements mimétiques et les sentiments suscités par des situations critiques". Par contre, les

1 A titre d'exemple, le Britannique Pete Goss a récupéré Raphaël Dinelli sain et sauf. Philippe Jeantot, organisateur du Vendée Globe : "Raphaël a prouvé ses capacités pour se lancer dans cette aventure... J'ajoute que le sang-froid avec lequel il a mis en route deux balises de détresse, puis une troisième quelques heures plus tard avant de l'éteindre, mercredi 25 décembre, montre que Raphaël a des qualités de marin qui dépassent la norme. Alors qu'il était en panne de moyens de transmission dans la tempête, il a ainsi voulu nous indiquer qu'il était en vie après son incident, puis qu'il avait repris le contrôle de la situation pour attendre les secours. J'ai d'ailleurs demandé jeudi aux autres concurrents d'utiliser la même méthode pour communiquer avec nous dans une telle situation" (C. DE ChENAY, Le Monde, 28 déc. 1996, p. 15).

2 La sélection des traits de caractère retenus par Goffman n'est guère expliquée, elle est intéressante, mais présente le défaut d'être trop arbitraire.

3 Cf. N. ElIAS, E. DUNNING, op. cit., p. 106.

4 Selon l'expression utilisée par Goffman pour qualifier les relations entre ordre de l'interaction et ordre social. E. GofFMAN, "The Interaction Order", American Sociological Review, vol. 48, n 1, Feb. 1983, pp. 1-17. 
singularités culturelles influencent davantage les narrations de la presse sportive.

\section{La lisibilité et la simplicité des récits sportifs les prédisposent à s'adapter aux logiques marchandes}

Le succès des commentaires sportifs doit certainement beaucoup à la simplicité de leurs schémas actanciels. Les expériences sportives proposent le plus souvent un cadre très simple : une opposition entre les locaux et les visiteurs ou entre le champion national et des étrangers. Les résultats ne souffrent pas d'ambiguité, les classements sont faciles à réaliser et la différence entre le bon et le mauvais est aisée. Même si la rationalisation apportée par le marketing conduit à une certaine uniformisation des spectacles, le théâtre, la littérature ou le cinéma présentent fréquemment des personnages et des situations plus complexes. La simplicité et la lisibilité des affrontements sportifs s'intègrent bien dans une logique de massification des spectacles qui va de pair avec une simplification des personnages et des problèmes; la diversité, les nuances ou les subtilités des personnages laissent souvent place à des oppositions beaucoup plus tranchées entre deux camps (les " bons" et les " méchants").

Une des forces du récit sportif est qu'il se prête facilement à la rationalisation et au calcul. Les journaux sportifs abondent de données sur les rencontres (nombre de tirs, vitesse des balles, temps de possession du ballon, etc.) et de discours techniques. Par rapport à d'autres formes de récit, la narration sportive occupe une place tout à fait particulière puisqu'elle réussit à mêler rationalité et émotion, répondant ainsi à des demandes narratives plutôt masculines. Cette simplicité et prévisibilité des événements sportifs les prédisposent à jouer un rôle dans les rationalisations marchandes et expliquent leur attrait particulier. Il est plus facile de vendre des espaces publicitaires et d'assurer des taux d'audience aux investisseurs grâce aux spectacles sportifs. La moindre efficacité marketing d'événements, tels que les guerres ou faits divers, provient de leur caractère trop aléatoire ${ }^{1}$; même s'ils présentent des opportunités pour les médias, leur gestion économique est plus difficile alors que les événements

1 Qui, par ailleurs, ne peuvent que faire rarement l'objet d'émissions spécifiques. Le cynisme et la rationalité économique (attente d'audience) pourraient cependant conduire à construire des événements. 
sportifs s'intègrent facilement dans les moments les plus profitables (prime-time) des grilles télévisuelles et s'intègrent bien dans les plans marketing.

\section{La trame des commentaires sportifs}

Chaque article sur le sport présente une cohérence propre mais il entre également dans la cohérence globale des journaux et de narration des événements. Le cadrage de l'action avec une mise en tension ${ }^{1}$, les régulations émotionnelles, les analyses des conduites et la sélection de ce qui doit être conservé en mémoire sont des éléments qui structurent fréquemment les articles.

Le cadrage de l'action entre dans la composition de nombreux commentaires sportifs, notamment dans les discours rituels sur les grands événements qui célèbrent les lieux, les personnes et les institutions. Une partie du récit journalistique se structure autour du cadrage temporel et spatial de l'événement. Il y a une phase de préparation, qui présente le décor et les acteurs ${ }^{2}$, dans laquelle on présente l'ordre "normal" des choses et où l'on célèbre, réifie et probablement fait

1 Voir C. Pociello, Les cultures sportives, Paris, PUF, 1995, p. 143.

2 Les journalistes décrivent les acteurs, la ritualisation des rencontres et les déférences qui suscitent le respect et la sacralisation mutuelle des adversaires. Par exemple : "Même Cédric Pioline, avec ses airs de dilettantes, s'est piqué au jeu. Depuis un an, Yannick Noah a "apprivoisé" ce joueur sauvage et solitaire qui admet un goût très modéré pour les échanges verbaux, au changement de côté comme en conférence de presse. D'abord méfiants l'un envers l'autre, les deux hommes ont appris à se respecter. Ensemble, ils travaillent presque sans parler" (P. JOLLY, Le Monde, 12 févr. 1996, p. 17). Ou encore : "Le géant néo-zélandais a écumé les mers les plus dures du globe, où il a collectionné les records. Aujourd'hui, il est en passe de devenir le deuxième marin, en cent quarante-quatre ans, à arracher l'Aiguière d'argent aux États-Unis. Douze ans plus tard, le 7 avril 1995 à San Diego, les deux marins les plus célèbres des antipodes se sont rencontrés pour la première fois dans l'intimité. A la veille des régates de la finale de la Coupe Louis-Vuitton, qui devaient désigner le vingt-neuvième challenger de la Coupe de l'America, Peter et Pippa Blake avaient répondu à l'invitation à dîner de John et Rasa Bertrand. Avant de s'affronter, les deux responsables des Défis néozélandais et australien avaient trouvé cette occasion de s'avouer leur respect mutuel, mais aussi de mieux mesurer tout ce qui les différencie, au-delà de leur passion commune pour la voile". (G. ALBouY, "Peter Blake, gentleman aventurier de l'America", Le Monde Horizons-Enquête, 11 mai 1995, p. 12). 
exister, des valeurs sportives. Généralement, le cadre ${ }^{1}$ de l'expérience sportive et ses enjeux sont définis dans le début de l'article.

Durant l'action, le commentaire est essentiellement descriptif, explicatif, emphatique. Le spectacle sportif diffusé par la télévision est abondamment narré en direct. Les émotions, les conduites, les enjeux ou les attitudes font partie des préoccupations des réalisateurs ou des commentateurs du direct ; à leur manière, ils font du spectacle un récit. Le commentaire écrit est moins emphatique. Il constitue une autre forme de narration qui semble compléter le commentaire à chaud. L'analyse du contenu des textes journalistiques nous permet de retenir deux axes importants d'organisation des discours : la régulation des émotions et le rappel des manières légitimes d'agir. Les commentaires se finissent souvent par un bilan qui permet de décrypter l'action, de tirer les leçons morales, de préparer les rencontres suivantes. Lorsqu'un événement est important, on retrouve toutes ces dimensions ; sur des commentaires plus ponctuels, l'une ou l'autre dimension peut être absente. La diversité des journaux, des rubriques, des styles journalistiques et des pratiques sportives font que ces éléments peuvent se décliner de différentes façons ${ }^{2}$.

\section{Un cadrage de l'action}

Même si l'image est déjà une façon de traiter la réalité, il semble qu'elle ne suffise pas toujours ; commenter ou redire les événements est important pour beaucoup de téléspectateurs. La majorité des lecteurs d'articles sportifs regardent également les spectacles. Le texte journalistique semble répondre à une demande supplémentaire

\footnotetext{
1 Au sens de Goffman (Les cadres..., op. cit.). Le travail de cadrage d'une activité visant à répondre à la question que chacun se pose face à une situation ordinaire : "que se passe-t-il ?". Lorsque les situations sont habituelles la question ne se pose pas, l'acteur comprend immédiatement ce qui se passe ; les événements sportifs importants font l'objet d'un travail de cadrage pour, d'une part, stimuler l'intérêt pour une rencontre et, d'autre part, l'issue des interactions étant incertaine, pour tenter d'anticiper le déroulement des confrontations.

2 Nous illustrerons nos propos à partir de différents extraits d'articles en privilégiant le traitement d'une cérémonie sportive importante dans un journal (l'Euro 1996 traité par Le Monde) et en étayant avec d'autres références (diversité de journaux et de sports).
} 
d'explication ${ }^{1}$, de décodage et de mise en ordre des événements ${ }^{2}$. Le support écrit n'est pas que redondant par rapport à la perception du spectacle.

L'engouement pour le spectacle sportif peut s'expliquer à la fois parce qu'il s'appuie sur des actions qui ne sont pas inscrites sur le registre de la fiction et qui ont des conséquences réelles, et par la possibilité pour le spectateur de retraiter les situations par rapport à ses propres expériences sociales ; on peut y voir le courage, le mérite, le cran, etc. ${ }^{3}$ A la fois modèle d'identification qui facilite projection (grâce notamment à son rôle dans une logique d'affirmation identitaire $^{4}$ ), et repère dans la compréhension du monde social. Le discours sportif a du sens parce qu'en parlant de la logique et du déroulement des actions dans le sport, il renvoie à la compréhension d'actions situées dans d'autres cadres sociaux ${ }^{5}$. Travail, souffrance, liens sociaux, économie, politique... peuvent être évoqués par le biais des discours relatifs au sport.

La Coupe d'Europe de football de 1996 nous fournit une illustration de ce que peut être un travail de cadrage de l'action. Il s'agit ici de donner de l'intelligibilité aux situations à venir et

${ }^{1}$ Les lecteurs réguliers de $L$ 'Équipe sont aussi ceux qui aiment le sport à la télévision (sondage TF1-BVA, 1984).

2 Le sens de la mise en ordre ne peut conduire à faire des journalistes des défenseurs systématiques des dominations sociales établies. Il existe un journalisme critique qui bouscule parfois l'ordre social. Par exemple, la diversité des commentaires conduit à des représentations contradictoires de la féminité (R. M. PIRINEN, "The Construction of Women's Position in Sport: A Textual Analysis of Articles on Female Athletes in Finish Woman Magazines", Sociology of Sport Journal, $\mathrm{n}^{\circ} 14$, 1997, pp. 290-301). Même si l'autorité institutionnalisée des médias donne un caractère officiel à leur version de la réalité (voir E. GoFFMAN, "L'ordre de l'interaction", in Y. WINKIN, Les moments et leurs hommes, Paris, Éd. du Seuil/de Minuit, 1988, pp. 207 et 230), la remise en ordre est aussi à comprendre dans le sens d'une recherche de cohérence et d'une "interprétation des individus".

3 Par exemple, J.-L. Godar perçoit dans les images une valorisation du travail ; il déclare que "au sport on voit des gens au travail, surtout maintenant qu'il est professionnel. Même s'ils gagnent des milliards, on peut se sentir l'égal des champions, parce qu'on voit ce travail" (entretien diffusé sur LCI, cité par C. FrEY, "Les forçats du bitume", Le Nouvel Observateur, Télé O, n² 201, 5-11 juillet 1997). Cette manière de percevoir le sport rappelle des valeurs traditionnellement associées au travail : l'effort et la souffrance. Le sport offre un moyen de rendre lisible et de rappeler ces liens alors que l'effort dans le travail a perdu de sa visibilité : les transformations du travail ne permettant plus très souvent d'associer de la même façon travail et effort.

4 Voir C. BROMBERGER, Le match de football, ethnologie d'une passion partisane, Paris, MSH, 1995.

5 Voir E. GoFFMAN, Les cadres..., op. cit. 
d'organiser les attentes vis-à-vis de l'événement. Le cadre de l'interaction est présenté dans la préparation de la cérémonie. Les propriétés des interactants ${ }^{1}$, leurs ambitions ${ }^{2}$, les risques des rencontres ${ }^{3}$ y sont définis de façon à anticiper les conduites probables et de mieux saisir les décalages entre les conduites attendues et les conduites effectives ${ }^{4}$. On ne peut faire bonne figure ou perdre la face qu'en référence à ce qui peut être attendu ${ }^{5}$. Il y a une définition préalable des enjeux des rencontres et une mise en intrigue. On note également une célébration des lieux ${ }^{6}$ et une définition des territoires ${ }^{7}$. Le cadrage $s^{\prime}$ accompagne de références et de rappels relatifs aux identités des communautés qui s'affrontent ${ }^{8}$ et la dramatisation ainsi que les discours emphatiques de mise en intrigue sont très présents ${ }^{9}$.

\section{Une régulation des émotions}

Même si les questions relatives au dopage ou à l'argent suscitent nombre d'articles, le journalisme sportif ne porte pas majoritairement sur des analyses critiques ou complexes du sport ${ }^{10}$. De nombreux articles décrivent ou suggèrent des émotions ${ }^{11}$. Des affrontements

1 "Le football français achève invaincu sa préparation à l'Euro 96", Le Monde, 7 juin 1996, p. 21.

2 P. CEAuX, "Les «Boys» de Terry Venables rêvent d'un couronnement à domicile", Le Monde, 8 juin 1996, p. 20.

3 P. DE BEER, "Mobilisation générale de la police anglaise", ibid.

4 P. CEAuX, "Face aux Turcs, les Croates flottent dans leurs habits de favoris", $L e$ Monde, 13 juin 1996, p. 20, ou B. HoPQUIN, "L'Allemagne telle qu'on l'attend et telle qu'elle surprend", Le Monde, 18 juin 1996, p. 22.

5 B. Hopquin, "L'Euro 96 sera un test de santé pour le football européen", Le Monde, 8 juin 1996, p. 20.

6 P. BrousSARD, "Le vestiaire porte-bonheur de Wembley", Le Monde, 10 juin 1996, p. 19.

7 "L'Europe du football", Le Monde, 10 juin 1996, p. 1.

8 P. CEAUX, "Jordi Cruijff, fils de Johann, rend son identité au jeu néerlandais", Le Monde, 15 juin 1996, p. 22.

9 B. HopQuin, "La France retrouve la Bulgarie dans un match de tous les dangers", Le Monde, 18 juin 1996, p. 22 ; B. HoPQUn,, "L'Italie joue contre l'Allemagne sa survie dans l'Euro", Le Monde, 19 juin 1996, p. 24 ; P. CEAUX, "Le Danemark voit s'approcher l'abdication et l'exil", ibid.

${ }^{10}$ Le journalisme, en tant que produit de consommation, privilégie des récits assez simples dans lesquels le spectateur ou lecteur "consommateur" puisse facilement se projeter. Les dimensions "éducatives" du journalisme n'ont que peu de chance d'avoir une place importante dans des logiques marchandes.

11 Cependant, s'affrontent ici plusieurs conceptions du journalisme sportif avec une opposition entre le journaliste de télévision, qui doit réaliser un bon spectacle en direct et des analyses rapides durant des émissions sportives, et un journalisme 
collectifs aux exploits individuels, en passant par la résistance aux éléments naturels, les sports permettent d'offrir une diversité de situations génératrices d'émotions. L'intérêt pour le spectacle sportif est vif parce qu'il met fréquemment en scène les plaisirs et les douleurs de champions qui, de manière dominante, sont des hommes et des femmes ordinaires, souvent "du peuple", et parfois de cultures différentes ${ }^{1}$. Ainsi les commentaires sur des personnages importants ou les héros du jour, joueurs ${ }^{2}$ ou entraîneurs ${ }^{3}$, abondent.

Le commentaire sportif dans la presse prépare et fait revivre des émotions tout en jouant sur la signification du spectacle ${ }^{4}$. La mise en jeu du corps et de valeurs à travers l'action, accentue les possibilités pour un spectateur de vivre intensément les situations; le spectacle crée de l'émotion dans une existence où le registre émotionnel est plus contrôlé. Le commentaire sportif participe ainsi au processus de régulation des émotions en fournissant " un cadre imaginaire destiné à provoquer une excitation imitant celle de la vie réelle, tout en évitant ses dangers et ses risques" 5 . On comprend mieux que la rationalisation marchande du sport se love parfaitement dans l'hypertrophie émotionnelle et intimiste du loisir dominant que constitue la télévision ${ }^{6}$.

d'analyse et de critique qui se rapproche davantage des formes plus classiques du journalisme.

1 Voir A. EHRENBerg, Le culte de la performance, Paris, Calman-Lévy, 1991, et également J. S. LEITE LOPES et S. MARESCA, op. cit.

2 P. CEAuX, "L'Angleterre place ses espoirs dans les pieds de Paul Gascoigne", Le Monde, 18 juin 1996, p. 22 ; P. Georges, "Gascon de Gascoigne", Le Monde, 28 juin 1996, p. 30 ; P. CEAuX, "Vaclav Nemecek, un Tchèque en version tricolore", Le Monde, 26 juin 1996, p. 21 ; B. HoPQUN, "L'apprentissage italien de Christian Karembeu", Le Monde, 22 juin 1996, p. 22.

3 B. HopQuin, "Les petites contrariétés d'Aimé Jacquet", Le Monde, 24 juin 1996, p. 17 ; P. Ceaux, "Miroslav Blazevic, l'entraîneur croate, en quête de revanche", ibid.

4 L'analyse des enregistrements radiophoniques des spectacles de boxe atteste aussi du rôle important que la radio a pu jouer dans la mise en forme et en tension du spectacle sportif. Voir A. RauCH, "Épistémologie d'une histoire du spectacle radiodiffusé", in Sport, relations sociales et action collective, Talence, MSH d'Aquitaine, 1995.

5 N. Elias, E. DUNNING, op. cit., p. 54, tout en ayant une fonction "mimétique" et "cathartique".

${ }^{6}$ Ce qui ne correspond pas à une tendance isolée ; les sociétés qualifiées de postmodernes valorisent l'intensité émotionnelle des spectacles (G. DEBORD, La société du spectacle, Paris, Champ libre, 1971) et dans la plupart des consommations (Z. BAUMAN, Imitation of Postmodernity, London, Routledge, 1992); les centres commerciaux ressemblent à des centres de loisirs; les parcs d'attraction à des grands magasins, des clowns animent ou décorent les fast-food (G. RTTZER, op. cit.) 
Ainsi, la préparation, la suggestion ou le rappel de l'émotion est souvent une dimension essentielle des propos. Les commentaires des photos, comme le texte principal, sont l'occasion d'une abondance d'adjectifs qui suggèrent différents registres affectifs (joie, colère, souffrance, peur, douleur, courage...). Il y a une organisation précise des émotions selon le type de victoire et la qualité des adversaires ; les conduites émotionnelles doivent rester en relation avec la dimension de l'événement ${ }^{1}$. Les commentaires décrivent et définissent une façon légitime de réguler des émotions qui scandent les contrôles émotionnels établis.

Une partie de l'intérêt des commentaires provient de la facilité avec laquelle ils peuvent être associés à d'autres expériences émotionnelles importantes de la vie quotidienne. Ils renvoient à des personnages auxquels le lecteur peut s'identifier et à des événements proches de son quotidien tels que des erreurs, accidents, réussites, séparations, rencontres, naissances, déclins, ascensions, etc. D'autres récits portant sur le sport suivent des logiques proches du commentaire journalistique. C'est le cas des commentaires publicitaires des marques de sport qui exploitent abondamment le registre émotionnel. Par exemple, pour toucher les consommateurs, P. Knight, PDG de la marque Nike, s'appuie sur les émotions sportives et les possibilités d'empathie du sport en mettant en scène une sorte de proximité intimiste avec les athlètes ${ }^{2}$. Les références à d'autres situations sont fréquentes et le passage d'un registre à un autre, par exemple de l'ambiance de fin de rencontre à une ambiance triste de fin de vacances $^{3}$, favorise les possibilités d'empathie. La fatalité des actions

et la télévision est une télévision de l'intimité (D. MEHL, La télévision de l'intimité, Paris, Éd. du Seuil, 1996).

1 "Tout à coup, les Français sont revenus sur la pelouse, en petites foulées, titulaires et remplaçants mêlés. Pour un tour d'honneur? L'initiative aurait été prématurée. Une victoire contre l'Australie (2-0) en match d'ouverture ne saurait justifier pareil débordement" (J. BOzONNET, "Les footballeurs français s'économisent", Le Monde, 23 juillet 1996, p. 16).

2 Il déclare que "To create a lasting emotional tie with consumers, we use athletes repeatedly throughout their careers and present them as a whole people. So that consumers feel that they know them. It is not Charles Barkley (NBA star) saying buy Nike shoes, it's seeing who Charles Barkley is... We win their hearts as well as their feet" (cité par N.K. DENZIN, "Dennis Hopper, McDonald's and Nike", in B. SMART (ed.), Resisting McDonaldizition, London, Sage, 1999).

3 "Les sacs de sport étaient déjà entassés dans le hall de l'hôtel. Le chauffeur du car commençait à faire tourner son moteur. Quelques joueurs attardés répondaient encore aux questions des journalistes. Une pluie d'été commençait à tomber sur la région de Wigan... Les adieux français à l'Euro 96, jeudi 27 juin, ressemblaient à 
sportives rappelle la vulnérabilité de la vie et des expériences sociales qui peuvent basculer facilement de l'euphorie de la victoire à la "dysphorie" de l'échec".

\section{La narration rappelle les manières légitimes d'agir}

Ainsi, la narration sportive est plus qu'un bavardage; elle est souvent à la fois une description des manières d'agir et d'interagir et se constitue en tant que référent à l'action. Elle met aussi en scène des systèmes de justification de l'action. Le bavardage sportif n'est pas neutre, il a tendance à rappeler les codes sociaux pour justifier ou critiquer des conduites. Il exerce parfois une violence symbolique qui contribue à faciliter le maintien de l'ordre. A travers des remarques déférentes, qui semblent insignifiantes, sont rappelées les manières légitimes de penser et d'agir. Par ses nombreuses déférences et sa mise en scène d'une sorte d'unité symbolique, la ritualisation sportive (présentation des équipes, cérémonies d'ouverture et de clôture, remise des prix...) renforce la légitimité, voire fait exister, des groupements sociaux ${ }^{2}$. La célébration de la nation (en France, le défilé sur les Champs-Élysées) est une manière de la faire exister et de constituer son unité en minimisant son hétérogénéité et en masquant ses tensions. Les commentaires sur les attitudes des "personnalités" -les émotions des stars du show-business ou l'enthousiasme des hommes politiques- mettent à la fois en scène une unité sociale, le peuple et l'élite vivant des émotions identiques, et rappellent l'importance des personnages dominants. En effet, parler de l'enthousiasme des "personnalités" (les gros plans, photos et commentaires sur

ces fins de vacances grises et tristes, quand tout le monde se quitte sans trop savoir si les promesses de retrouvailles seront tenues ou non (P. BROUSSARD, "La préparation de la France pour la Coupe du Monde 1998 risque de souffrir de l'exode des meilleurs joueurs", Le Monde, 29 juin 1996, p. 26).

1 La voix est triste, l'homme abattu. Reynald Pedros ne peut raconter son cauchemar qu'en murmurant. Ce penalty manqué, il le chuchote. Il le confesse. Comme une mauvaise action dont on se repentira toute une vie. Cela va si vite. Un face à face d'une seconde avec Petr Kouba, le gardien tchèque, et le destin d'un match, le sort d'un joueur, basculent. Reynald Pedros n'a pas encore trouvé la force d'avoir pitié de lui-même. Il se déchire en des mots durs, définitifs. Ils lui sont venus à l'esprit, dès l'arrêt de Kouba. «J'ai tout de suite pensé que c'était moi qui avait éliminé l'équipe de France», dit-il" (P. CEAUX, "Confessions sur deux penalties manqués", Le Monde, 29 juin 1996, p. 26).

2 Les nationalismes ont abondamment puisé dans les rituels sportifs pour se mettre en scène. 
Jacques Chirac exultant lors de la victoire de l'équipe de France en Coupe du monde de football) constitue une marque de déférence qui rappelle les dominations sociales et la division sociale des représentations ; leur joie ostentatoire les rapproche des hommes ordinaires, accentue l'importance de l'événement et cautionne la légitimité de la liesse populaire.

Les personnages connus ou les grands événements n'ont pas l'exclusivité des marques de déférence. Plus généralement, le commentaire sportif sert de support à un propos sur la cohérence de nos actions et aussi sur les conditions de félicité de nos actes. Le récit tente de donner du sens aux conduites qui se déroulent dans le cadre du sport. Il opère une mise en ordre symbolique en jouant de diverses façons sur les liens entre conduites et codes. Les commentaires donnent de l'intelligibilité aux règles sociales qui organisent nos comportements. L'efficacité symbolique du sport, en tant que modèle de référence pour d'autres conduites de la vie quotidienne, la doit à ses caractéristiques propres et à sa médiatisation. Les codes ne sont évidemment pas spécifiques aux activités sportives; le sport est néanmoins devenu un référent. Il rappelle, fixe, déplace ou modifie des codes comportementaux à travers des spectacles sportifs qui mettent en scène de nombreuses séquences d'action et de décision et dans les conduites narrées par les journalistes ${ }^{1}$. Par exemple, le "manque de réalisme des joueurs" est une thématique qui revient fréquemment pour exprimer l'idée qu'il faut adapter ses actions à la situation, à ses prétentions et aux attitudes des adversaires ${ }^{2}$. Le commentaire sportif prend d'autant plus de sens qu'il peut renvoyer à de nombreuses autres situations de la vie quotidienne. A l'image des manuels de savoir-vivre ${ }^{3}$, les commentaires des conduites sportives rappellent, en les réactualisant, les codes sociaux qui organisent la trame de notre action quotidienne ${ }^{4}$. Les conduites sportives servent à

1 Les décisions doivent sembler logiques, justifiables ou critiquables. La coexistence avec autrui implique de rendre compréhensibles nos conduites. Dans les interactions quotidiennes, comme dans les discours sur les actions humaines, il y a une nécessité de mise en cohérence et de justification. Une grande partie du travail de la presse sportive vise à justifier et à mettre en cohérence des actions.

2 La formule résonne comme une critique des utopies et rappelle des croyances qui sont au fondement des idéologies libérales.

3 Voir D. PICARD, Les rituels du savoir-vivre, Paris, Éd. du Seuil, 1995.

4 Les commentaires de la responsable de l'agence de mannequins Marylin -qui a créé un département chargé de sélectionner des sportifs pour des campagnes publicitaires- traduisent assez bien ces liens. L'agence ne recrute pas pour la "plastique" du sportif, mais en fonction de "l'image positive qu'il [le sportif] dégage et de son 
expérimenter les qualités humaines nécessaires aux actions situées dans d'autres cadres sociaux. Les liens symboliques sont même parfois exploités économiquement ${ }^{1}$. Souvent, les commentaires sont associés à des références aux manières légitimes de faire; ainsi tel journaliste peut décrire les règles de déférence et les codes qui organisent les interactions dans le milieu tennistique ${ }^{2}$. La conception de l'ordre interactionnel dépend ici de la nature et de la distribution du capital symbolique dans l'espace sportif. Le journaliste rappelle que seule la performance durable donne une légitimité aux joueurs et il expose un exemple de profit symbolique (m'entraîner avec eux, et ça signifie beaucoup). Le journaliste sélectionne et commente des extraits des déclarations de E. Kafelnikov qui explique que les gestes et paroles autorisés -les conduites- renvoient à la fois aux règles générales qui organisent les interactions sociales et aux particularités dues à leur inscription dans l'espace sportif. Ainsi le commentaire suggère une définition des postures (s'il ratatine instinctivement sa haute taille) et des attitudes (l'air vaguement inquiet) que le lecteur peut retrouver dans les nombreuses circonstances de la vie quotidienne où le passage d'une situation à l'autre, d'un espace à un autre, implique une adaptation des dispositifs comportementaux (par une sorte d'effet de seuil symbolique, on se tutoie ou on réajuste son apparence).

L'exemple de E. Kafelnikov n'est pas atypique ; le journalisme sportif, plus que dans d'autres rubriques journalistiques, met en scène

attitude tant lors des compétitions que dans la vie quotidienne" (cité par la Lettre de l'économie du sport, 4 mars 1998).

1 Parmi les nombreux exemples de la littérature qui associe les qualités dans le sport et la vie professionnelle, C. Schmaltz, consultant et formateur spécialisé dans les techniques de développement professionnel et personnel, déclare, dans un ouvrage consacré aux vertus éducatives du golf, que : "le golfeur doit prendre conscience de ses comportements, apprendre à observer et à tirer profit des succès et des erreurs, bref, exercer des qualités indispensables à la vie professionnelle réussie" (C. SCHMALTZ, Les leçons du golf, Paris, Éd. Charlie Mike, 1997).

2 "Aujourd'hui numéro 7 mondial, il est ravi «d'être devenu quelqu'un». Même s'il ratatine instinctivement sa haute taille (1,90 mètre) et prend toujours l'air vaguement inquiet lorsqu'on l'interroge. Conscient de sa valeur intrinsèque, Evgueni Kafelnikov a longtemps considéré qu'on ne lui accordait pas la déférence méritée. En remportant en 1994 les trois titres qui l'ont hissé aux portes du Top 10, il a forcé le respect. Maintenant, Sampras et Stich me parlent, disait-il fièrement à l'automne de cette même année. Ils me demandent de m'entraîner avec eux, et ça signifie beaucoup" (P. JOLLY, "Evgueni Kafelnikov hisse la Russie en finale de RolandGarros", Le Monde, 10 juin 1996, p. 18). 
des codes d'honneur et de déférence facilement identifiables ${ }^{1}$. Ce n'est de loin pas un monopole, les rituels diplomatiques décrits dans la presse renvoient également aux impératifs de faire bonne figure ou de sauver la face. Cependant, le commentaire sportif s'appuie sur des situations plus proches des réalités quotidiennes des lecteurs; les fréquents manquements aux règlements dans la vie quotidienne impliquent habituellement une réparation des offenses. Bien que beaucoup plus ritualisées et plus affirmées, les logiques de l'honneur exposées dans les commentaires sont proches de celles des interactions sociales du quotidien. L'exemple d'une inconduite du pilote automobile $M$. Schumacher rend compte de la dramatisation. L'incident fait la "une" des journaux ${ }^{2}$ alors qu'il semble dérisoire par rapport à d'autres événements. Ce sont à la fois des nécessités économiques (le drame fait plus vendre que les événements insignifiants) et le rôle de référent joué par la conduite des sportifs qui expliquent l'intérêt porté à ce non-événement. Cet ensemble de règles non respectées permet de rappeler l'existence de certains codes sociaux. En effet, les conduites déférentes font souvent partie des dispositifs de sacralisation de la personne et, à travers elle, de la collectivité et de son fonctionnement. Dans ce cas, ce n'est pas le non-respect des règles des compétitions de la Fédération Internationale des Sports Automobiles qui dérange le plus, mais le non-respect des codes d'honneur. A travers son action, l'Allemand M. Schumacher a mis en jeu l'honneur de Ferrari et de l'Italie.

Les commentaires portent aussi sur l'absence d'harmonie entre les conduites et les attentes sans nécessairement faire référence à un

1 L'analyse du contenu (sur la même période) des articles portant sur la musique rock dans le journal $L e$ Monde, atteste d'une singularité du sport. Quelques exemples: "L'aventure européenne du Nîmes Olympique a pris fin, jeudi 31 octobre en Suède, avec les honneurs. Les «crocodiles» se sont imposés (1-0) sur le terrain de l'AIK Stockholm", "FootBalL, Coupe des coupes, Nîmes avec les honneurs", Le Monde, 2 nov. 1996, p. 14 ; "Cette saison, trois des quatre équipes engagées hors des frontières ont fait honneur à la patrie" (B. HoPQuIN, Le Monde, 30 oct. 1996, p. 20) ; "Ce baroud s'avérait insuffisant. Nîmes pouvait s'offrir un tour d'honneur inattendu en terre hongroise" (B. HoPQuIN, Le Monde, 28 oct. 1996, p. 24); "Virtuellement éliminés, les Bretons ne pouvaient que tenter de sauver l'honneur" (Le Monde, 26 oct. 1996, p. 21) ; "Et pourtant, il s'en est fallu d'un rien, mardi 24 septembre, pour que les joueurs du Bayern de Munich n'entament un tour d'honneur" (S. Joby, Le Monde, 12 oct. 1996, p. 20).

2 "Et maintenant Schumacher des excuses", titre sur 8 colonnes du quotidien sportif Corriere dello Sport (27 oct. 1997). "Schumacher s'est couvert de honte et doit être chassé sans attendre par Ferrari (éditorial de L'Unità, 27 oct. 1997). 
événement précis. De nombreux articles font référence à des catégories sociales construites -les anciens du sport, les jeunes, les sportives, les fondateurs- et aux décalages entre les conduites et les attentes. Dans un article sur la cycliste $\mathrm{J}_{\text {. Longo }}{ }^{1}$, le journaliste, tout en réaffirmant la règle traditionnelle du respect pour les anciens, fait écho aux regrets de la cycliste concernant l'absence de respect de l'ancienneté dans le cyclisme féminin. Dans le cas de J. Longo, une plus grande déférence est attendue dans les interactions quotidiennes. Le journaliste condamne la forte domination masculine sur les sports "virils" qui conduit à une faible reconnaissance des performances féminines. Les logiques de l'espace sportif permettent d'expliquer que la notoriété (d'une femme dans un sport populaire masculin) ne suffit pas à constituer un capital symbolique convertible économiquement.

Les controverses et critiques ne peuvent masquer une certaine tendance de la rhétorique sportive à réaffirmer des valeurs traditionnelles. Les articles rappellent avec une grande régularité la normalité des comportements masculins et féminins. L'association entre force, courage, héroïsme, caractère, volonté et les hommes est dominante. La pratique des femmes ne met pas en cause les représentations traditionnelles de la féminité puisqu'elle est très associée aux sports qui valorisent la forme gestuelle plutôt que l'efficacité de l'action (patinage, gymnastique, natation synchronisée...) $)^{2}$. En commentant les conduites sociales, les journalistes rappellent aussi la hiérarchie des valeurs. Par exemple, l'honneur n'est pas forcément à celui qui gagne : la retraite du joueur de tennis S. Edberg est à la fois l'occasion de commémorer une carrière, de valoriser davantage un temps important de la vie sociale (la retraite), de montrer que la retraite mérite davantage de déférence que la victoire et d'indiquer un usage

1 "Jeannie Longo aura trente-huit ans le 14 octobre, mais se refuse à les faire. S'il est adage que le sport conserve, il est également avéré que la haute compétition n'a aucun respect pour les anciens. «La» Longo devrait être depuis longtemps en dépassement d'âge, surclassée, blasée, si ce n'est usée... Paradoxalement, une des sportives les plus populaires de France, avec Florence Arthaud et Marie-José Pérec, n'est toujours pas parvenue à inspirer le respect. Même avec le temps, les ricanements que suscite le cyclisme féminin font toujours mal. «Quand je m'adresse à des services de communication d'entreprise pour trouver un sponsor, je suis souvent prise de haut», raconte Jeannie Longo" (B. HopQuin, Le Monde, 11 oct. 1996, p. 23).

2 De nombreux travaux ont déjà permis de constater cela dans la pratique sportive. Voir notamment A. Davisse, C. LouveAu, Sports, école, société : la part des femmes, Joinville-le-Pont, Actio, 1991. 
adapté des émotions (le sourire de J.-P. Fleurian ${ }^{1}$ ) aux circonstances. Les codes d'honneur et le jeu des déférences réelles et attendues, sont aussi des façons de réaffirmer, et d'une certaine manière, de faire exister des catégories sociales. Les commentaires insistent volontiers sur le respect dû aux "anciens" (sportifs, dirigeants...) ; ce faisant, ils réaffirment une catégorie sociale et les codes sociaux associés (la déférence "normale" à l'égard des anciens) 2 .

\section{La construction de la mémoire des événements}

A l'issue du déroulement d'un événement, de nombreux articles répètent et prolongent les émotions, tirent des leçons pour les compétitions à venir et, pour les événements importants, proposent une sélection de ce qu'il faut garder en mémoire ${ }^{3}$. A travers le cadrage des événements (nombre de rencontres, victoires, faits remarquables...) et surtout les bilans, les journalistes construisent une histoire des sports. Une partie des articles est consacrée à la commémoration des événements et à une mise en ordre de l'histoire de la pratique. Les commémorations et glorifications sont à la fois des célébrations et des mises

1 "Il avait accepté que sa victoire, au tour précédent, soit confidentielle. Accepté que les honneurs reviennent à Stefan Edberg, dont il a précipité les adieux en Australie. Il ne s'était pas départi de son large sourire. Il comprenait. Il n'était pas le héros" (B. MATHIEU, "Les Internationaux de tennis : à Melbourne, Jean-Philippe Fleurian reste le seul Français en lice", Le Monde, 20 janv. 1996, p. 20).

2 L'exemple suivant, pris dans le tennis masculin, illustrera notre propos : "Le joueur russe a su respecter «l'ancien», Michael Stich, qui lui offrait la politesse de doubles fautes et surtout une victoire en trois sets dans un tournoi du Grand Chelem. Bien qu'insolemment chanceux avec les lignes, le Russe s'est appliqué plus ou moins consciemment à respecter «l'ancien»" (P. JOLLY, "Evgueni Kafelnikov entre dans la légende de Roland-Garros", Le Monde, 11 juin 1996, p. 20).

3 Par exemple: "Michel Platini tire les leçons de l'Euro pour la Coupe du Monde de 1998", Le Monde, ler juillet 1996, p. 16 ; "Premières leçons", ibid., p. 1 ; "La victoire du gardien de but", Le Monde, 28 juin 1996, p. 1. 
en ordre du passél. Pendant, et également après les rencontres, on observe une mise en perspective d'un point de vue historique ${ }^{2}$.

Les journalistes sélectionnent ce qui doit composer une anthologie sportive toujours utile à la célébration des valeurs nationales, locales ou individuelles auxquelles renvoie l'événement ${ }^{3}$; l'exégèse prépare les commémorations. A travers la manière de tirer des leçons, de construire une histoire spécifique des pratiques sportives et de rappeler, pour les commémorer, les éléments à retenir dans l'histoire de la pratique, les textes contribuent à façonner des identités sociales (individuelles ou collectives) ${ }^{4}$. C'est également la culture de l'événement, l'affût d'une performance exceptionnelle, la menace d'une série noire ou d'une malédiction comme la préparation de l'événement qui supposent de pouvoir disposer d'une mémoire importante. La mise en tension nécessite de rappeler les chronologies des victoires ou des défaites contre des équipes ou des hommes, lors de moments importants (coupe ou championnat) ou dans les lieux qui marquent le patrimoine du sport (Wembley, le Parc des Princes, etc.). Rappeler les séries, c'est savoir si les défaites malheureuses sont dues à la fatalité et si la chance ou le talent justifient les longues séries de victoires.

1 Par exemple : "René Lacoste, le dernier des Mousquetaires, autrement dit, pour les générations présentes, le dernier des Mohicans, est mort samedi à quatre-vingtdouze ans. Fracture du col du fémur, cancer de la prostate, bronchite chronique, toute une coalition aura dû se liguer pour emporter à cet âge respectable un homme dont on disait qu'il était de fragile constitution. Des générations entières de joueurs ont vécu ainsi dans l'ombre de ces vétérans, présents dans les mémoires et présents dans les tribunes, Borotra et Lacoste notamment, vigiles respectés de RolandGarros" (P. GeORGEs, "Les Crocodiles, aussi, meurent dans leur lit", Le Monde, 15 oct. 1996, p. 33).

2 P. Ceaux, "En ouverture, l'Angleterre se renferme dans son passé", Le Monde, 11 juin 1996, p. 19. Les lieux "historiques" sont également rappelés : P. BROUSSARD, "The Albert de Liverpool, archétype du «football pub»", Le Monde, 14 juin 1996, p. 22.

3 Par exemple, C. Montaignac, journaliste à L'Équipe, a intitulé une communication : "Constitution d'un imaginaire et d'une mémoire individuelle sur le sport grâce à certaines figures emblématiques" (Colloque "montrer le sport", Paris, INSEP, mars 1999). Les douze images emblématiques qu'il a sélectionnées (uniquement des hommes) jouent, selon lui, un rôle important dans la constitution de l'histoire du sport.

4 B. HopQuin, "L'Italie a imposé son collectivisme face aux individualités russes", Le Monde, 13 juin 1996, p. 20. 


\section{Diversité de l'offre narrative}

De très nombreux articles présentent des dimensions descriptives, commémoratives ou emphatiques dans lesquelles des thèmes tels que l'équipe, le collectif, le record, les métaphores guerrières ou l'héroïsme sont très souvent exploités. La grande diversité du contenu des commentaires révélée par l'analyse remet en cause les visions unitaires du spectacle sportif. A partir des analyses lexicales, nous pouvons observer des différences significatives entre les sports : le tennis, le cyclisme, le football et la voile ne valorisent pas des conduites identiques. L'analyse quantitative nous donne des indications assez claires bien que très superficielles. Par exemple, les commentaires sur le football valorisent à la fois les dimensions collectives de l'action, son inscription dans les enjeux internationaux et les acteurs institutionnels du football (président, dirigeants, entraîneurs...). Le cyclisme souligne davantage les traits de caractère (courage, ténacité, panache...), la voile est l'occasion d'une abondante narration sacralisante de la mer et dans les commentaires du tennis transparaissent quantité de déférences personnelles et de codes d'honneur. La diversité des pratiques sportives permet de constituer une offre narrative très étendue. La distribution du contenu des commentaires confirme le pluralisme des ancrages des discours sur le sport et donc la possibilité d'intéresser un très large public masculin. La diversité des commentaires dépend aussi de la position du journal dans le champ médiatique. Les différences de traitement de chaque événement rendent lisible la malléabilité des sports qui se voient déclinés et interprétés selon les propriétés des journaux et la manière dont les journalistes cherchent à toucher des publics ${ }^{1}$.

1 Par exemple, cette malléabilité se lit dans la manière dont la presse féminine traite le sport : les aspects techniques des sports sont négligés au profit d'une narration plus intimiste (vie de famille, amour, biographie des sportifs). Le commentaire agit sur la signification du spectacle. Quand, pour des raisons marketing, la NBC a fait le choix de cibler les commentaires d'un match de boxe (JO 1996) sur les femmes et les familles (en faisant davantage porter les narrations sur l'intimité des protagonistes plutôt que sur des aspects techniques ou statistiques), l'audience s'est fortement féminisée et fut composée à $50 \%$ de femmes adultes contre $35 \%$ d'hommes (T. Miller, A. McHoul, Popular Culture and Everydaylife, London, Sage, 1998). L'accentuation de la concurrence entre les chaînes de télévision en France implique déjà de jouer sur la signification du spectacle pour élargir le 
L'homologie entre le type de commentaires et les propriétés socioculturelles des lecteurs contredit les prétentions universalistes du spectacle sportif ${ }^{1}$; par exemple, les émotions semblent se décliner selon la diversité des propriétés des lecteurs ${ }^{2}$. Quel que soit le type de journal, les commentaires rappellent des règles d'action et d'interaction ; cependant, la forme et le contenu des articles traitant du sport varient selon plusieurs paramètres (les lignes éditoriales, les auteurs, les sports, le type d'événement). On peut également noter d'importantes différences au sein de chaque quotidien selon les rubriques. Le sport peut être traité en privilégiant la description (les résultats commentés sont dominants) ou en favorisant des récits (rubrique Horizons dans Le Monde, par exemple). Le traitement des composantes émotionnelles dépend des journaux ${ }^{3}$. L'Équipe valorise d'abord les émotions collectives par un discours emphatique associé à de nombreuses images qui mettent en scène les gestes les plus significatifs des combats et des exploits. Dans L'Équipe, les rationalisations techniques (scores des joueurs, temps de passage, notes, etc.) font écho aux emphases émotionnelles de la "Une" ou aux notes humoristiques des titres. A l'opposé, Le Monde fait une économie de photos et privilégie des formes plus littéraires de la description de l'événement. L'exemple de la victoire de la France en Coupe Davis est révélateur de cette distribution. $L$ 'Équipe valorise la consécration par le lieu et la commémoration de la culture du tennis ${ }^{4}$. L'Humanité insiste sur les

public. Par exemple, on intègre une présence féminine (S. Thalman, ex Miss France) dans une émission sportive traditionnelle pour développer des commentaires moins techniques au profit d'anecdotes et de menus propos sur la vie quotidienne, l'intimité et la vie affective des sportifs.

1 Cela confirme l'aspect ténu des liens entre spectacles sportifs et cultures, plusieurs types et niveaux de lecture du spectacle direct ou télévisuel étant possibles à partir d'émotions proches. Par contre, les formes des narrations sportives sont fortement liées aux propriétés des lecteurs. Au-delà de nos quelques indications, une étude de la manière de lire les textes ou de percevoir les images permettrait probablement de mieux observer les clivages sociaux qui sont masqués par les célébrations sportives.

2 L'étude des événements et des personnages du sport qui marquent la mémoire, montre que les émotions sportives dépendent de paramètres socio-démographiques tels le sexe, l'âge et la profession. La diversité sociale résonne dans la diversité des figures héroïques du sport. Voir P. DURET, L'héroïsme sportif, Paris, PUF, 1994.

${ }^{3}$ La variation des surfaces rédactionnelles consacrées aux photos est un bon indicateur. Par exemple, la photo des aérostiers B. Piccard et B. Jones (qui ont réalisé le tour. du monde en ballon) représente $67 \%$ de la surface de la première page de L'Équipe, $39 \%$ de celle de France Soir, $10 \%$ de celle du Figaro et de L'Humanité et moins de $2 \%$ de celle du Monde (journaux datés du lundi 22 mars 1999). 
dimensions sociales de l'événement ${ }^{1}$ et Libération souligne les manières de faire hétérodoxes de Y. Noah ${ }^{2}$. Le Figaro et Le Monde se contentent de titres beaucoup plus sobres ${ }^{3}$ et France Soir s'appuie sur l'image et la référence à la cérémonie du 14 juillet $t^{4}$.

Le traitement des événements sportifs reflète souvent les préoccupations d'une collectivité ; les journalistes en tant qu'acteurs sociaux semblent les mettre en scène à travers une construction sociale du spectacle. En sélectionnant les disciplines ${ }^{5}$, les événements, les images, les commentaires ou les champions qui méritent d'être interviewés, les journalistes font un usage social et économique d'une offre de spectacles qui met en scène des appartenances sociales dans leurs composantes subjectives (tribus sportives rassemblées autour d'une passion) et dans celles qui paraissent les plus objectives (sexe, âge, nationalité). Le journaliste compose un texte à partir d'une sélection arbitraire d'éléments ; il met en scène une réalité reconstruite, les référents culturels établis sont retraités à partir de leurs propres croyances. Sans pour autant inventer une réalité, le journaliste a tendance à imposer des représentations sociales ou sa propre vision de l'événement ${ }^{6}$.

4 P. Marlat, "Les champs de la victoire", L'Équipe, 4 déc. 96 ; un sous-titre valorise le défilé des Champs-Élysées et Roland-Garros pour rendre hommage aux "Mousquetaires".

1 B. VINCENS, "Yannick Noah : «J'essaie de donner aux joueurs ce que je n'ai pas reçu», L'Humanité, 5 déc. 96.

2 C. Losson, "Noah fait son show après la victoire. Des vestiaires au pince-fesses, choses vues et entendues dimanche soir à Malmö", Libération, 3 déc. 96.

3 "Lendemains de triomphe. La conquête de la Coupe Davis par la France devrait logiquement dynamiser le tennis national. Attendons de voir", Le Figaro, 5 déc. 96 et "Les vainqueurs de la Coupe Davis sont fêtés à Paris", Le Monde, 5 déc. 96.

4 "Comme un 14 juillet", France Soir, 4 déc. 96.

5 On retrouve dans les journaux des différences de distribution des sports homologues à celles des chaînes de télévision. Même si l'opposition entre service public et offre marchande n'existe pas, on remarque une opposition entre une plus grande valorisation des pratiques collectives dans les journaux comme L'Équipe, France Soir et les quotidiens régionaux et une place plus importante (en proportion) des pratiques individuelles, telle que la voile, dans les journaux plus associés aux fractions culturelles comme Libération et Le Monde.

${ }^{6}$ Les journalistes contribuent à diffuser des représentations sociales : la marchandisation du football anglais des années 90 est largement soutenue par la presse qui suggère de traiter le spectateur comme un consommateur et non plus comme un fan de football (voir A. KING, "New Directors, Customers, and Fans : the Transformation of English Football in the 1990s", Sociology of Sport Journal, $\mathrm{n}^{\circ} 14,1997$, pp. 224-240). E. DUNNING, op. cit., remarquait également que la possibilité pour que les interventions des chercheurs en sciences sociales soient réellement prises en compte est pratiquement nulle à la télévision et dans les 
Le langage journalistique n'est donc pas seulement une des formes de représentation du sport, il renvoie également à des représentations des conduites sociales. Il y a, à la fois, des représentations au sens théâtral -des mises en scène- et des mises en ordre d'un point de vue symbolique. Les athlètes, en tant que symboles sociaux (d'une nation, d'une région, d'une ville, d'une classe sociale, d'un sexe...) ne mettent pas en jeu leurs seules caractéristiques personnelles. A travers leurs caractères, ils affirment les qualités de leur groupe de référence $^{1}$; leur personnalité est emblématique, elle peut être considérée comme une parcelle de "mana"2. En parlant des héros et en contribuant à leur fabrication, le journaliste manipule des symboles sociaux ; sous couvert de sport, il décrit des personnes qui interagissent. Le récit sportif ne présente pas de grandes singularités par rapport à d'autres formes de communication dans l'usage de métaphores ${ }^{3}$; s'il s'écarte singulièrement d'autres rhétoriques, c'est par sa capacité à donner du sens à des conduites situées dans d'autres cadres sociaux et à répondre aux demandes de référents symboliques en offrant un spectre assez large de métaphores de la vie sociale ${ }^{4}$.

tabloïds et un peu plus grande dans des journaux de "qualité" comme le Guardian, le Times et l'Independant.

1 Parmi les nombreuses analyses qui attestent de ces processus, on peut citer le travail de Tony Masson sur Stanley Matthews, footballeur, qui fut d'abord un symbole régional avant de devenir un symbole national. Artisan et fin technicien du football, il savait se comporter en gentleman en dehors des terrains et, de ce fait, obtenir une large popularité auprès du public (T. MASSON, "Stanley Matthews, la genèse d'un symbole", Actes de la recherche en sciences sociales, $\mathrm{n}^{\circ} 103$, juin 1994, pp. 62-69).

2 E. DURKHEIM, Les formes élémentaires de la vie religieuse, Livre II, Paris, PUF, (2e éd.), 1990.

${ }^{3}$ Le langage est métaphorique et le langage journalistique semble avoir généré "un système caractéristique de représentation métaphorique" ; voir J.-P. MEUNIER, "Métaphores journalistiques", Recherches en communication, $\mathrm{n}^{\circ} 2$ 2, 1994, p. 66.

4 Voir C. BROMBERGER, op. cit. 